\title{
Optical coherence tomography characterizes the roughness and thickness of the heterogeneous layer on cortical bone surface induced by Er:YAG laser ablation at different moisture contents
}

\author{
Wenyan Huang', Chudan Gao ${ }^{2}$, Yintao Lan ${ }^{2}$, Sujuan Zeng ${ }^{1}$, Janak L. Pathak ${ }^{1}$, Miao Zhou ${ }^{1}$, Lihong Ge ${ }^{1,3}$, \\ Jian Zhang ${ }^{1,2,4}$
}

${ }^{1}$ Key Laboratory of Oral Medicine, Guangzhou Institute of Oral Disease, Affiliated Stomatology Hospital of Guangzhou Medical University, Guangzhou 510140, China; ${ }^{2}$ Department of Biomedical Engineering, School of Basic Medical Science, Guangzhou Medical University, Guangzhou 511436, China; ${ }^{3}$ Department of Pediatric Dentistry, Stomatology Hospital of Peking University, Beijing 100081, China; ${ }^{4}$ State Key Laboratory of Respiratory Disease, National Clinical Research Centre for Respiratory Disease, Guangzhou Medical University, Guangzhou 510120, China

Correspondence to: Jian Zhang, PhD. Department of Biomedical Engineering, School of Basic Medical Science, Guangzhou Medical University, Guangzhou 511436, China. Email: jianzhang@gzhmu.edu.cn.

Background: As an innovative bone ablation tool, pulsed erbium: yttrium-aluminum-garnet (Er:YAG) laser is being used in clinical practice. It faces the same problems as traditional tools: the water content of bone usually changes with the position, while the amount of water spray in the process of laser irradiation is also uncertain. Real-time monitoring of the effects of laser bone ablation is necessary, but effective tools are still lacking. In this study, we examined the feasibility of rapidly and non-destructively evaluating the surface properties of bone after Er:YAG laser irradiation at different moisture contents by optical coherence tomography (OCT).

Methods: Bone specimens $(n=90)$ collected from pig cortical bone were used in this study. All bone specimens had similar volume and surface characteristics after machining. To display the baseline level before Er:YAG laser ablation, a control group $(n=10)$ without dehydration or Er:YAG laser ablation was examined with OCT, scanning electron microscopy (SEM), and energy dispersive X-ray (EDX) spectroscopy. The remaining specimens were randomly divided into four groups ( $\mathrm{n}=20 /$ group) with different moisture content: dried (Group 2 and Group 4) or not dried (Group 1 and Group 3). Pulsed Er:YAG laser (120 mJ/pulse, $20 \mathrm{~Hz}, 100 \mu \mathrm{s}$ ) was used for perpendicular irradiation with (Group 1 and Group 2) or without (Group 3 and Group 4) water spray. The treated specimens were subjected to SEM, EDX spectroscopy, OCT, and then processed for histological evaluation.

Results: After Er:YAG laser ablation, the melting effect was obvious in the SEM results of the dry groups and non-sprayed groups (Groups 2-4). EDX spectroscopy showed that the content of calcium and phosphorus and their ratios remained unchanged in the undried and with water spray group (Group 1) were the closest to the control group. Three-dimensional (3D) OCT could evaluate the depth and shape of ablation grooves. The roughness of the laser-ablated surface could be visualized by extracting the surface lines from cross-sectional OCT images. The results illustrated that the laser ablation with water spray could achieve a smoother surface. Furthermore, OCT results demonstrated that a layer with high image intensity was generated on the bone surface after laser irradiation. The thickness of these layers showed a correlation with whether or not the laser irradiation was sprayed with water. Histology showed that thin eosin-stained layers were created in all experimental groups, which matched well with OCT results of the layers with high image intensity. No denatured layer was observed in the non-irradiated areas of bone tissues.

Conclusions: OCT could rapidly and non-destructively visualize the bone surface before and after Er:YAG laser ablation at four different moisture contents. The morphology of ablation grooves, as well as the roughness and thickness of the heterogeneous layer on the bone surface, could be characterized quantitatively with good correlation with SEM and histology. This study will promote the development of 
OCT as an efficient and accurate tool for evaluating laser ablation of bone.

Keywords: Bone; Er:YAG laser; ablation; optical coherence tomography (OCT); roughness; heterogeneous layer

Submitted Jul 18, 2019. Accepted for publication Feb 18, 2020.

doi: $10.21037 /$ qims.2020.02.15

View this article at: http://dx.doi.org/10.21037/qims.2020.02.15

\section{Introduction}

There is a long history of using manual instruments such as metal drills for hard-tissue procedures in oral and maxillofacial surgery. However, using these instruments is usually accompanied by extremely harsh noise, and even worse, could cause the deposition of metal shavings (1). In recent years, the laser, which can achieve cutting effects similar to conventional rotary instruments, was introduced into dentistry as an alternative to traditional mechanical instrumentation for the removal of biological tissues $(2,3)$. The erbium: yttrium-aluminum-garnet (Er:YAG) laser emits a 2,940 $\mathrm{nm}$ wavelength that coincides with the absorption peak of water and is also well absorbed by the hydroxyl groups in hydroxyapatite (HA) crystals. Its mechanism in hard tissue ablation is the thermo-mechanical effect induced by the "micro-explosion" of water molecules (4). The sudden evaporation of bound water causes microexplosions that blast away tiny particles of bone tissue (5). Because of its many advantages, such as precise cut geometry, high bactericidal effect, reduced tissue bleeding, and less noise, vibration, and more discomfort for the patient than rotary instruments, the Er:YAG laser has been reported as suitable for use in clinical bone surgery (6-8). Compared to conventional rotary instruments, the Er:YAG laser can promote early bone healing due to high levels of platelet-derived growth factor released in the wound and the creation of a favorable surface for cell attachment $(9,10)$.

Real-time and non-destructive monitoring of ablation efficiency and morphology on bone tissue surface is essential in clinical practice. The current mastery of ablation efficiency depends on the physician's experience and visual observations. Traditional methods to evaluate bone surfaces, such as histology and scanning electron microscope (SEM), are mostly destructive tests and cannot be directly applied in clinical cases $(11,12)$. Therefore, it is necessary to develop a testing aid that can evaluate the character of bone tissue in a real-time and non-invasive manner. Optical coherence tomography (OCT) is a realtime and noninvasive optical imaging modality based on the back-scattered signal intensity from within structure $(13,14)$. OCT has a high resolution, significant depth, fast speed, and three-dimensional (3D) imaging ability (15). Its applications in dentistry include assessing dental caries and detecting tooth-composite interfacial integrity and tooth cracks (16-19). In addition, OCT also has unparalleled security and convenience. Endoscopic OCT devices are being used for clinical cardiovascular and bronchial imaging, and hand-held OCT devices for retinal and tympanic membrane imaging are being developed (20-22). Therefore, it is possible to develop OCT oral imaging equipment.

Laser irradiation may cause thermal tissue damage, which is closely related to the internal water content of the tissue and external water spray. It is known to all that a great difference exists between the water content of bone dense matter and that of bone loose matter. Moreover, inadequate water irrigation may occur during laser irradiation in clinical situations, and irradiation without water-cooling may have detrimental effects on bone tissue. Therefore, to ensure the clinical safety of Er:YAG laser during orthopedic surgery, a thorough analysis of the actual maximal thermal damage caused to the cortical bone in a clinical situation should be performed. In this study, four different moisture content models were constructed by changing internal water content and external water spray. Since cross-sectional or 3D images of specimens can be obtained by OCT, the purpose of this study was to examine the feasibility of rapidly and non-invasively evaluating the surface properties of cortical bone after Er:YAG laser irradiation at different moisture contents by OCT. SEM, energy dispersive X-ray (EDX) spectroscopy, and pathological section were also used in this study. Our findings may help to develop a testing aid that can evaluate the thermal damage of bone tissue in a real-time and non-invasive manner.

\section{Methods}

\section{Specimen preparation}

The experimental specimens were freshly isolated cortical 
bones from pig tibias. The surrounding soft tissues and periostea were removed, making the specimens as smooth as possible. All of the collected cortical bone specimens were cut into several pieces with a low-speed electric saw under running water to obtain regular bone specimens with size $5 \times 6 \mathrm{~mm}^{2}$. The thickness of all specimens was the original size of the pig bone, ranging from 3 to $5 \mathrm{~mm}$. Each bone specimen was observed under a stereo microscope, and then any cracked bone pieces were discarded. All of the bone specimens were stored in the $0.9 \%$ physiological saline at $4{ }^{\circ} \mathrm{C}$ and used for experiment within one week. The specimens at room temperature were dried with compressed air before the experiment.

\section{Er:YAG laser ablation}

A commercially available pulsed Er:YAG laser (LightWalker, Fotona LLC., Dallas, USA) was applied for bone ablation. A sapphire tip $1.3 \mathrm{~mm}$ in diameter was used, and the laser tip was placed vertically on the bone surface. The laser beam propagated through the articular arm and was focused directly on the surface of specimens through the lens. The laser was set according to the recommended energy parameters $(120 \mathrm{~mJ} / \mathrm{pulse}, 20 \mathrm{~Hz}, 100 \mu \mathrm{s})$. Due to an energy loss of approximately $20 \%$ in the probe, the actual output energy at the tip was about $96 \mathrm{~mJ} /$ pulse, and the laser output energy density was approximately $0.36 \mathrm{~J} / \mathrm{mm}^{2}$. With or without continuous water spray and $90 \%$ air pressure, the laser transmission was carried out by R14 handpiece in the indirect contact mode from the target $1 \mathrm{~mm}$, and the laser hand tool was moved to form a linear tunneling area through the full length of the bone specimens.

\section{SEM and EDX spectroscopy}

A back-scattering electron mode SEM (S-3400N II, Hitachii, Japan) was used in this study. The bone specimens were fixed with $4 \%$ paraformaldehyde solution for $48 \mathrm{~h}$, rinsed with water overnight, and then dehydrated using cryodesiccation. Then, the specimens were mounted, sputter-coated with gold, and observed with SEM at 50, 250 , and 500 magnifications. The accelerating voltage was $15 \mathrm{kV}$. The composition of elements was detected by an EDX spectroscopy (550i, IXRF Sytems, Austin TX, USA) that was installed on the SEM. Each tested region was randomly selected from 3-4 laser-irradiated regions and non-irradiating regions to determine the contents of calcium ( $\mathrm{Ca}$, atomic \%), phosphorus (P, atomic \%), and carbon $(\mathrm{C}$, atomic \%). The ratios of calcium and phosphorus $(\mathrm{Ca} / \mathrm{P})$ were calculated from each region.

\section{The OCT system}

A spectral-domain OCT (SD-OCT) imaging system (Figure 1) was utilized in the study (23). This SD-OCT system utilized a single superluminescent diode (InPhenix, Livermore CA, USA) with an $840 \mathrm{~nm}$ central wavelength and an over $40 \mathrm{~nm}$ spectral bandwidth as the light source. A 50/50 broad bandwidth spliced fiber coupler (Ziyin Photoelectric Technology, Shenzhen, China) served as the core of the interferometer. The spectrometer of this OCT system integrated $1,200 \mathrm{~L} / \mathrm{mm}$ @ $840 \mathrm{~nm}$ volume phase holographic gratings (Wasatch Photonics, Logan UT, USA) and a 2,048-pixel line-scan camera (SF-10-02K40-GE-02, Luster Co., Ltd., Beijing, China). The spectrometer design was optimized for the light source of this system, and our SD-OCT imaging setup could achieve an axial resolution of $8 \mu \mathrm{m}$ and a lateral resolution of $12 \mu \mathrm{m}$ in the air. The imaging depth of this system could achieve around $4 \mathrm{~mm}$ for the clear optical phantom and about $2 \mathrm{~mm}$ for tissues such as muscle. The scan system adopted two high-speed galvo mirrors (Han's Motor, Shenzhen, China), which enabled rapid volume acquisitions at a high imaging speed of up to $18 \mathrm{kHz}$ A-scan per second. A custom C\# language code was developed for operating the SD-OCT system. As a result, the SD-OCT could carry out real-time, depthresolved, cross-sectional, and 3D imaging. The maximum viewing field of the SD-OCT system reached $10 \times 10 \times 2 \mathrm{~mm}^{3}$. Data acquisition and signal processing were simultaneously performed using a high-performance personal computer. A CCD camera (Daheng Optics, Shanghai, China) could live to monitor the application area and take photographs of the samples. OCT imaging could be performed along a path selected based on the photograph of a sample. With the help of the CCD camera, we were able to acquire the OCT images along with the same profile on the sample for each time point.

\section{Histological section}

The bone specimens in each group were fixed with $4 \%$ paraformaldehyde solution for $48 \mathrm{~h}$ and then were rinsed with water overnight. All bone specimens were soaked in EDTA decalcified solution for 21 days and flushed $4 \mathrm{~h}$ after decalcification. After dehydration, the specimens were embedded in paraffin and cut into $4-5 \mu \mathrm{m}$ tissue 


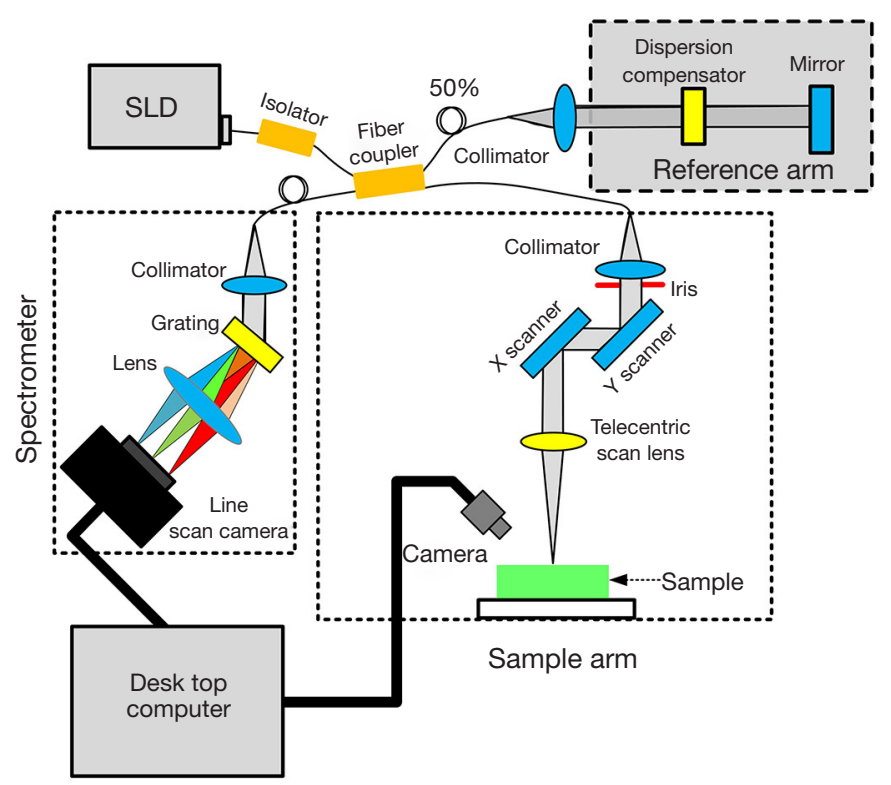

Figure 1 Schematic diagram of the spectral-domain optical coherence tomography imaging system.

sections. The specimens were stained with hematoxylineosin and subjected to light microscopy (DM4, Leica, Wetzlar, Germany). Photomicrographs were taken, and histomorphometric analyses were performed.

\section{Experimental protocol}

According to whether the specimens were dry and whether or not there was external water spray, the experimental specimens were divided into four groups: the undried and with water spray group (Group 1), the dry and with water spray group (Group 2), the undried and without water spray group (Group 3), and the dry and without water spray group (Group 4). Each group comprised of 20 specimens. Ten non-irradiating bone specimens were regarded as the control group. The drying method of placing the drying box at $60^{\circ} \mathrm{C}$ for $24 \mathrm{~h}$ was adopted. Then, the irradiated bone specimens were randomly distributed into two groups: the OCT-histology analysis group and the SEM-EDX analysis group. The OCT and EDX analysis were detected in the control group. The experimental protocols of this study are shown in Figure 2.

\section{Surface line and image profile extraction}

To acquire images with minimum inhomogeneity, imaging was performed multiple times in different regions. Images with the least heterogeneous presentation were imported and saved using MATLAB 8.0 (MathWorks, Natick MA, USA). OCT images were binarized and etched, and then surface lines could be extracted by a simple maximum detection method. To compare the roughness more accurately, all the surface lines were calibrated by the polynomial fitting. In addition, the OCT intensity profiles for each image were extrapolated according to the Hilbit envelope function. The OCT intensity profiles were determined on the basis of the distribution and density of the pixels within the image and provided information regarding the thickness of the bone tissues.

\section{Statistical analysis}

The differences in the calcium, phosphorus, and carbon content, as well as the groove depths on the surface of the bone tissues, were subjected to one-way analysis of variance (ANOVA) followed by the Bonferroni post hoc test for multiple comparisons at a $5 \%$ significance level $(\mathrm{P}=0.05)$. Differences in the ratios of calcium and phosphorus were analyzed by the Kruskal-Wallis test, and $\mathrm{P}<0.05$ was considered statistically significant. Quantitative data were expressed as mean \pm standard deviation. The calculations were handled using Prism 5.0 (GraphPad Software, San Diego CA, USA). 


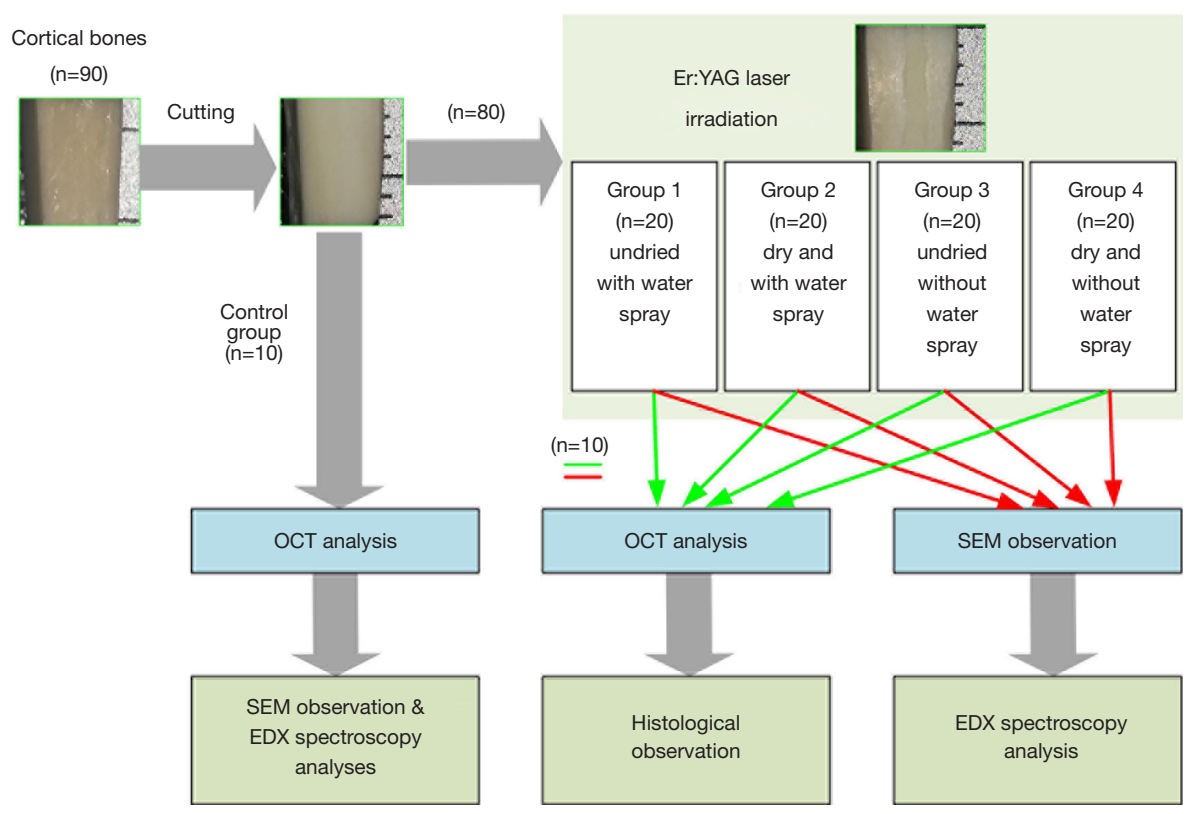

Figure 2 The experimental protocols of this study.

\section{Results}

\section{Performance of the OCT system}

The lateral resolution of the OCT imaging system depended on the bandwidth of light source that is shown in Figure $3 A$. The bandwidth of the light source is about $40 \mathrm{~nm}$. According to the calculation formula, $\mathrm{R}_{\text {axial }}=(2 \ln 2 / \pi) \times\left(\lambda^{2} / \Delta \lambda\right)$, where the $\lambda$ is the central wavelength, and the $\Delta \lambda$ is the spectral bandwidth, the axial resolution of the OCT system is $\sim 7 \mu \mathrm{m}$. Furthermore, the lateral resolution of the OCT system was measured by imaging a blade. The 3D OCT image of the blade is shown in Figure 3B. An OCT image profile was extracted and fitted to obtain a line expansion function. Normalized differentiation of the line spread function is shown in Figure 3. The full width at half maximum (FWHM) $\sim 12 \mu \mathrm{m}$ was the lateral resolution of the OCT system. We further imaged the bone tissue samples to test the imaging depth of the OCT system, as shown in Figure 3D. The OCT image was an $\mathrm{X}-\mathrm{Z}$ cross-sectional view that covered a $2 \times 2 \mathrm{~mm}^{2}$ area. The left part of the OCT image showed the location of the horizontal plane of bone, and the right part showed the inclined plane of bone. The imaging depth of the OCT system in bone was about $400 \mu \mathrm{m}$, as measured in the image by the green dash line. Based on these unique advantages, this OCT system is very suitable for the evaluation of laser- ablated bone surface.

\section{En-face micro-morphology of the bone surface observed by SEM}

The bone samples used in this study were machined. The first column of Figure 4 shows the SEM image of the bone without Er:YAG laser ablation. The trace induced by mechanical cutting could be clearly seen in the 50x magnification SEM image, as shown in Figure $4 A$. The surface of the bone tissue was very flat, but fine cracks could be seen in the high magnification SEM images (Figure 4B,C). The SEM images in columns 2-5 correspond to the bone tissue samples of the laser ablation experiments in Groups 1-4. Continuous grooves with clear and regular boundaries were observed on the surface of bone tissues in the 50× SEM image of Group 1 (undried and with water spray, Figure 4D). Under the condition of high magnifications (250x and 500x), a large number of multilayer protrusions were observed, which were slightly irregular and rough scales (Figure 4E,F). In Groups 2, 3 and 4 , the melting effect in bone tissues was obvious, and there were continuous grooves with irregular boundaries. Under the condition of high magnifications (250x and 500x), more irregular protrusions and micro-cracks were observed

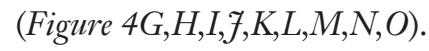




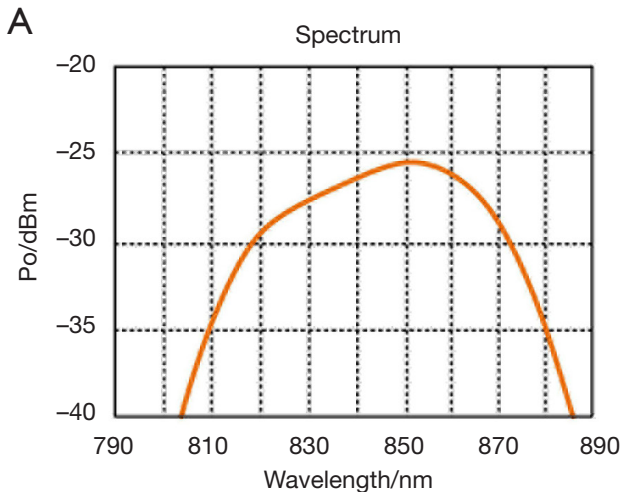

B
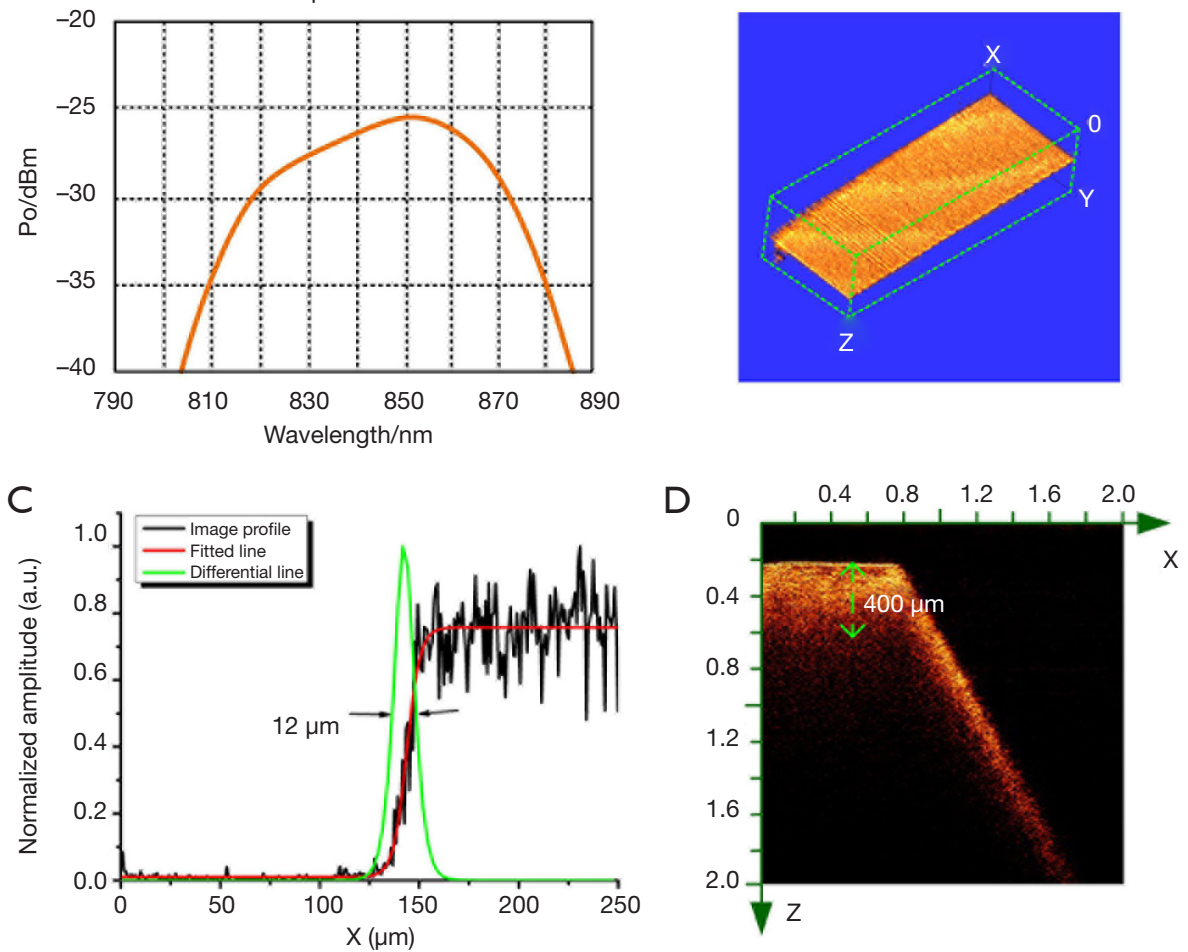

Figure 3 Performance of OCT system. (A) The bandwidth of light source used in the OCT system. (B) 3D OCT image of a blade. (C) OCT image intensity profile (black line), fitting line (red line), and corresponding normalized differential line (green line). (D) Crosssectional OCT image of a piece of normal cortical bone. OCT, optical coherence tomography.

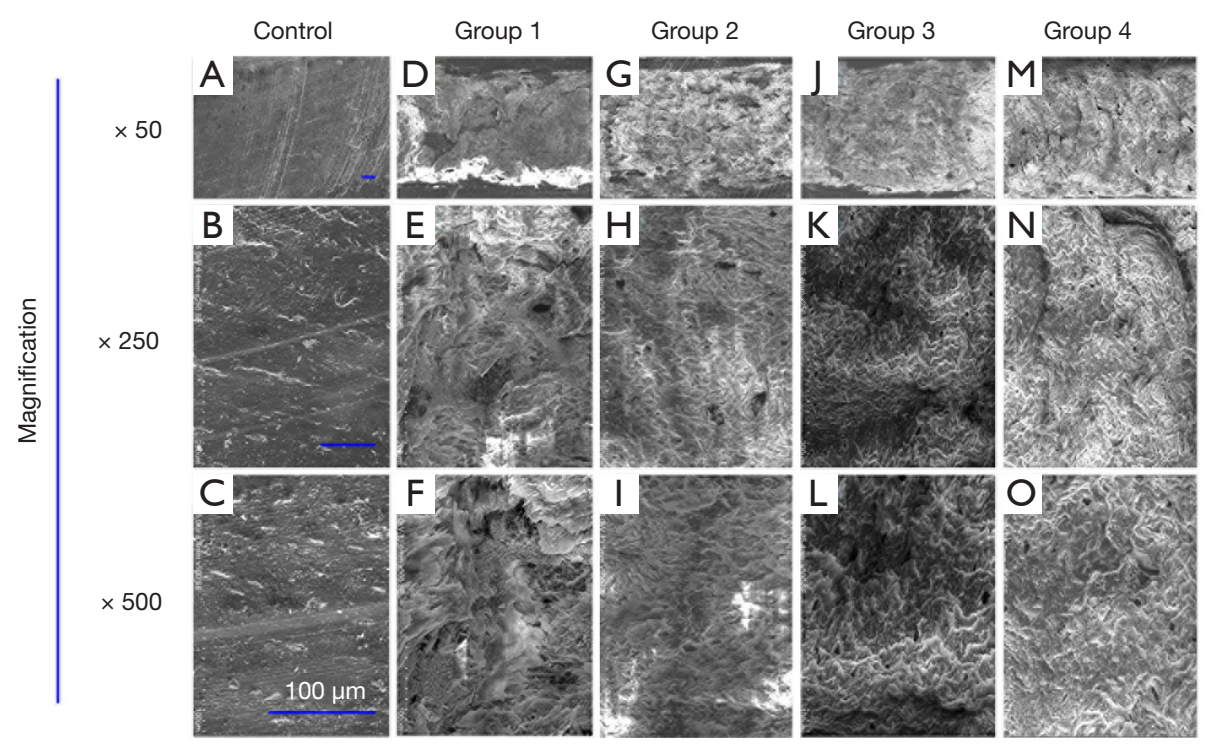

Figure 4 SEM images at three magnifications $(50 \times, 250 \times$ and 500x) used for characterizing bone surface before (A,B,C) and after Er:YAG laser-treatment at four different moisture contents [Group 1 (D,E,F), Group 2 (G,H,I), Group 3 (J,K,L), and Group 4 (M,N,O)]. Scale bars are $100 \mu \mathrm{m}$ in all images. 

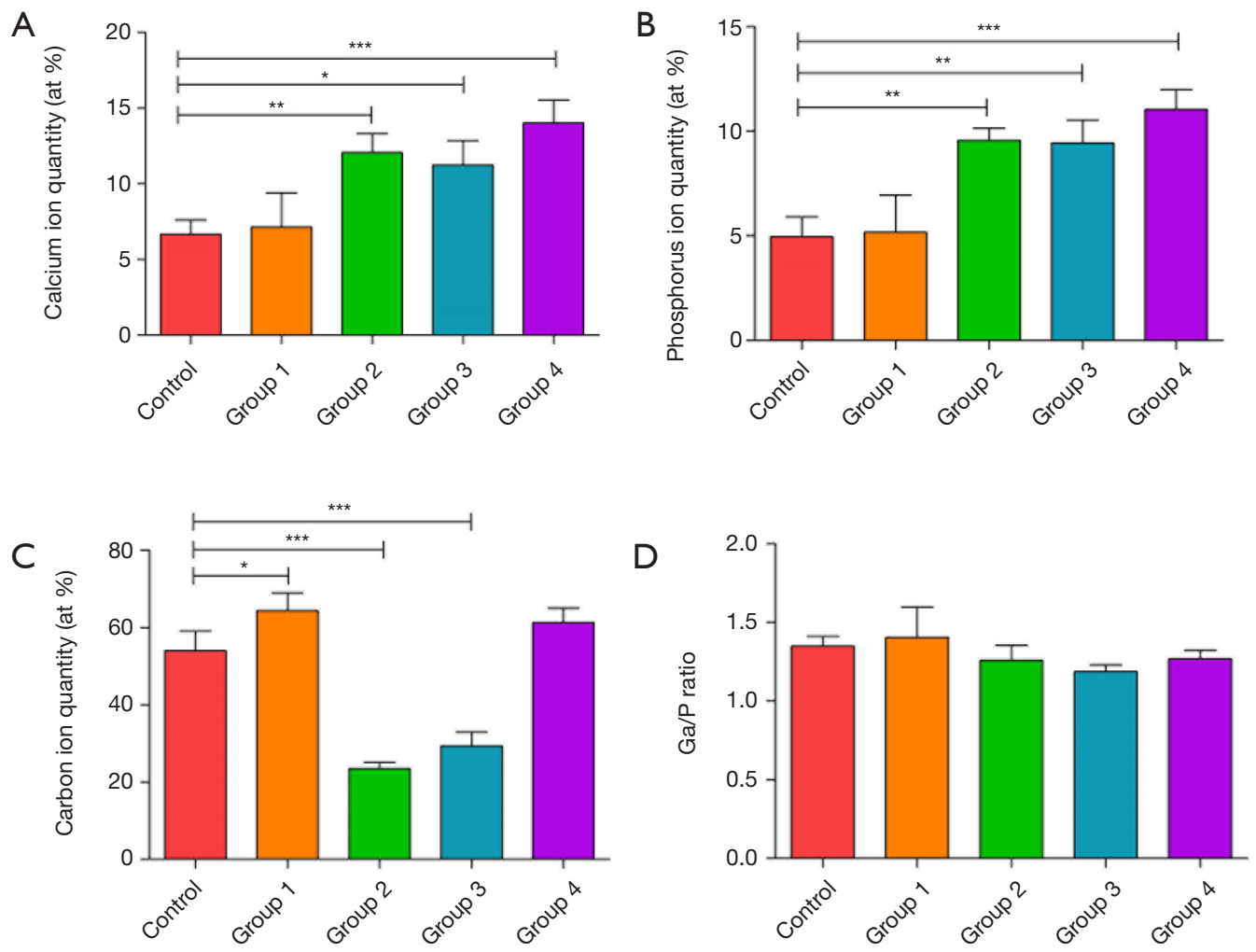

Figure 5 The calcium (A), phosphorus (B), and carbon (C) content on the surface of bone tissues after Er:YAG laser ablation at four different moisture contents (Groups 1-4) measured by EDX spectroscopy as well as the ratio between calcium and phosphorus (D). The control group represented the non-irradiated areas of bone tissue. * $\mathrm{P}<0.05,{ }^{* *}, \mathrm{P}<0.01,{ }^{* * *}, \mathrm{P}<0.001$.

\section{The elemental changes in bone surface assessing by EDX spectroscopy}

Calcium, phosphorus, and carbon are three key elements in bone tissue. The results of EDX spectroscopy analysis are shown in Figure 5. Compared to the control group (non-irradiating sites), the results from Group 1 (undried and with water spray) showed no significant changes in the amounts of calcium and phosphorus and $\mathrm{Ca} / \mathrm{P}$ ratios $(\mathrm{P}>0.05)$, except for slightly increased carbon component $(\mathrm{P}<0.05)$. The high water content and external water spray surface received more energy and reduced thermal deposition at the same time. Mechanical tissue was removed without undesirable thermal damage, leading to no significant change in calcium and phosphorus from the irradiated bone surface. In Groups 2, 3, and 4, the amounts of calcium and phosphorus were significantly increased compared with the control group, and Group $1(\mathrm{P}<0.05)$. A decrease in the amount of carbon was noted in Group 2 and Group $3(\mathrm{P}<0.05)$, while the carbon amount remained unchanged in Group $4(\mathrm{P}>0.05)$. The evaluation of the $\mathrm{Ca} / \mathrm{P}$ ratio showed no significant changes between the laserablated group and the control group $(\mathrm{P}>0.05)$. Once the water content of tissue is not high enough or without external water spray, no additional water is available for absorbing laser energy and the laser energy dries out the bone surface, resulting in an uncontrolled energy deposition and the consequent change of calcium and phosphorus from the irradiated bone surface.

\section{Evaluating the bone surface before and after Er:YAG laser ablation by $3 D$ OCT}

Figure $6 A$ is a brief introduction to the $3 \mathrm{D}$ OCT imaging process. As a non-contact imaging mode, the scans were $0.2 \times 4 \mathrm{~mm}^{2}$ and $2 \mathrm{~mm}$ in depth, centered on the laser-ablated bone surface with an $18 \mathrm{kHz}$ A-scan imaging speed. The acquisition time for a 3D OCT image was $\sim 2 \mathrm{~s}$. Figure $6 B$ 

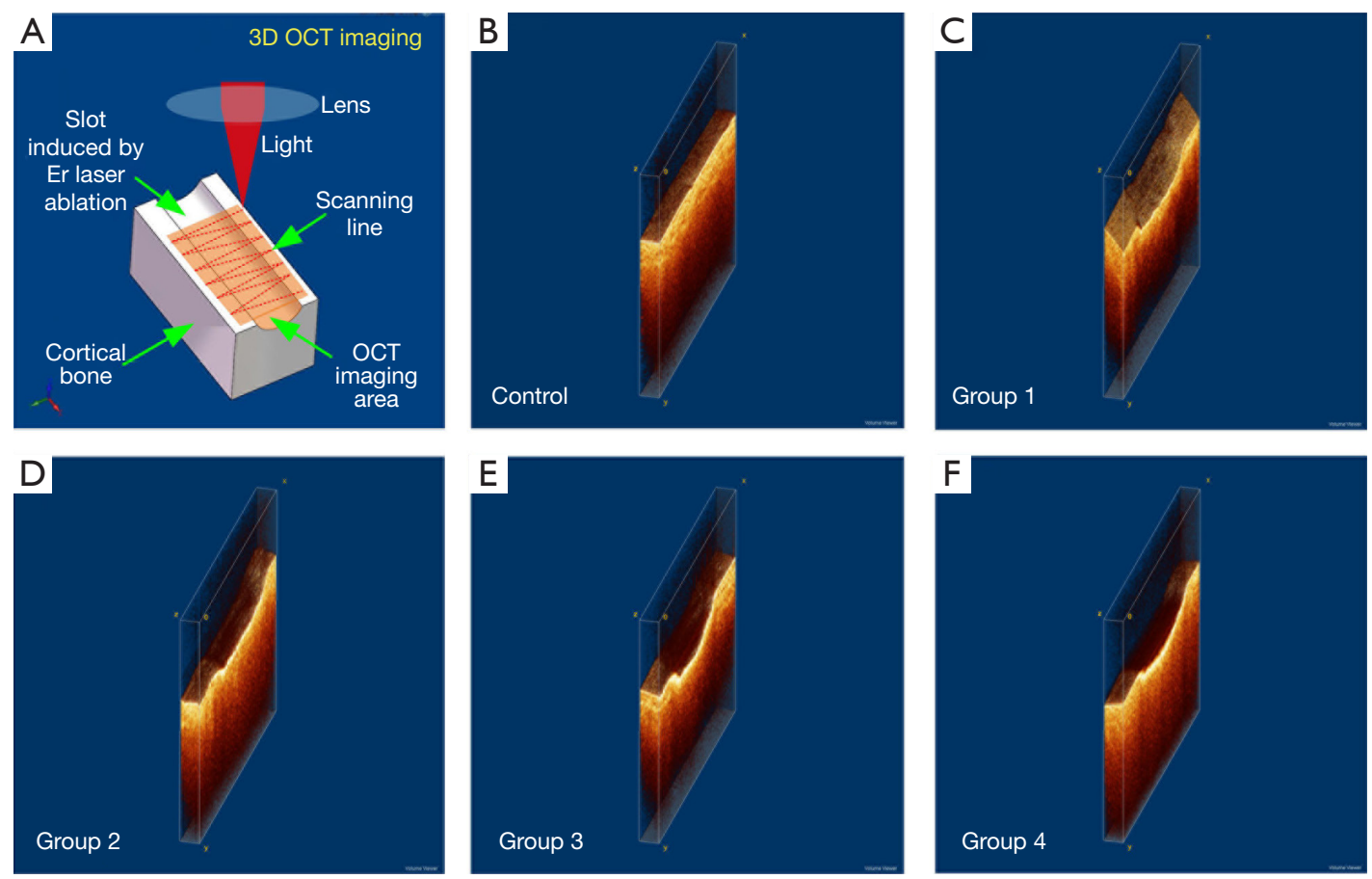

Figure 6 Evaluation of bone surface properties by OCT in 3D imaging mode. (A) Schematic of the imaging process. OCT 3D images of the bone tissues before (B) and after Er:YAG laser ablation (C,D,E,F) at four different moisture contents (Groups 1-4). OCT, optical coherence tomography.

shows the 3D OCT image of bone before Er:YAG laser ablation, depicting a very smooth surface. Figure $6 C, D$ show the $3 \mathrm{D}$ images of laser-ablated bone with water spray. Ablation grooves on the two bones were inverted trapezoid. Figure $6 E, F$ show the $3 \mathrm{D}$ images of laser-ablated bone without water spray. Ablation grooves induced without water spray showed a circular shape. The shape of the ablation groove depended on whether or not there was water spray. The groove depth was extracted from the OCT images and statistical analysis was performed, as shown in Figure 7. Among Groups 1-4, the highest ablation depth could be seen in Group $4(\mathrm{P}<0.05)$. The ablated depths of Group 1 were significantly lower than those of Groups 3 and $4(\mathrm{P}<0.05)$. There was no significant difference in groove depths between Group 1 and Group 2 ( $\mathrm{P}>0.05)$. These results demonstrated that OCT has the ability to evaluate ablation efficiency in real-time.

\section{OCT characterizing the laser-ablated surface of bone along the depth direction}

One of the significant advantages of OCT imaging is that it can obtain a high-resolution depth profile of samples.
Figure $8 \mathrm{~A}$ shows an OCT image of bone tissue in the control group. In this OCT image, the surface of bone tissue is smooth, with uniform light scattering characteristics in the depth direction. The green arrow indicated the boundary between bone tissue and air. Figure 8B,C,D,E show the OCT images of bone tissue treated with Er:YAG laser. The boundary of all four laser-ablated bones showed relatively high image intensity. The thickness of the high image intensity area was similar in Group 1 and Group 2, which were treated under water spray. The thickness of the high image intensity area increased when the bone was ablated without water spray. Group 4 showed the maximum thickness, which is related to having the lowest moisture content. There is an inevitable relationship between the high-intensity layer in the OCT images and Er:YAG laser ablation. The evaluation based on OCT image intensity was qualitative, and quantitative analysis based on FWHM will be given in the following section.

\section{Pathological verifying the heterogeneity of bone surface after laser ablation}

The Er:YAG laser-ablated bone specimens were further 


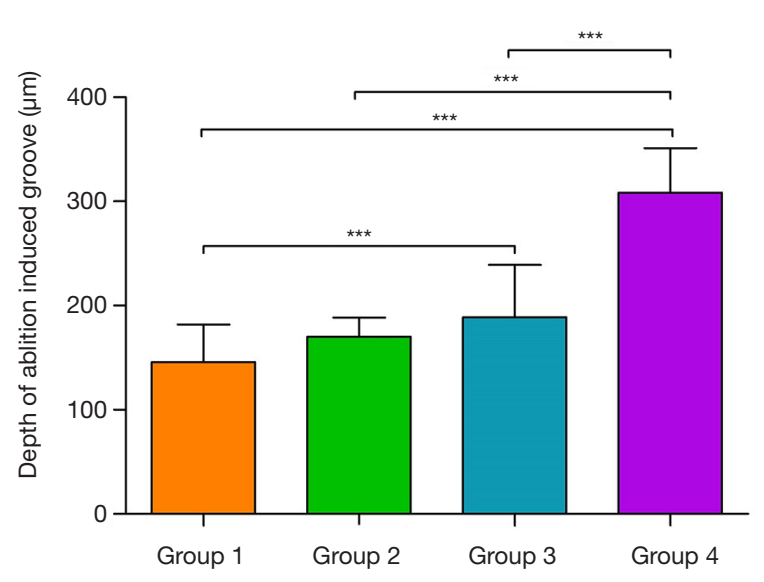

Figure 7 Quantitative evaluation of the groove depth induced by Er:YAG laser at four moisture contents (Groups 1-4) based on 3D OCT images. ${ }^{* *}, \mathrm{P}<0.001$. OCT, optical coherence tomography.

examined after sectioning and staining. The histological results are shown in Figure 9. The laser-irradiated bone areas are within the range of the black dashed lines. All groups had thin layers stained by eosin along the surface of the grooves with irregular boundaries and without a clear structure. The green arrows indicate the deep stained layers. The black arrows indicate the non-irradiated areas. No eosin-stained layer was observed in non-irradiating areas of bone tissues (Figure 9A,C,E,G). The eosin-stained layers in the non-sprayed groups (Figure 9E,F,G,H) were thicker than those in the water sprayed groups (Figure $9 A, B, C, D$ ). There was no significant difference in eosin-stained layers between the dry groups (Figure 9C,D,G,H) and the undried groups (Figure $9 A, B, E, F)$. The thickness of the thin eosin-stained layers was affected by the external water spray, but not by internal water content, which matches well with OCT results of the layers with high image intensity.

\section{Discussion}

Optical imaging technology and optical therapy technology play an increasingly important role in the process of disease diagnosis and treatment. The primary goal of this study was to test the feasibility of real-time evaluation of Er:YAG laser ablation on the cortical bone surface by OCT. At the same time, SEM, EDX, and pathology were also used in this study for qualitatively evaluating laser-ablated bone surface at four different moisture contents.

Holmes et al. obtained the range of thermal relaxation time in cortical bone are 400 to $500 \mu s$ (24). On the basis of

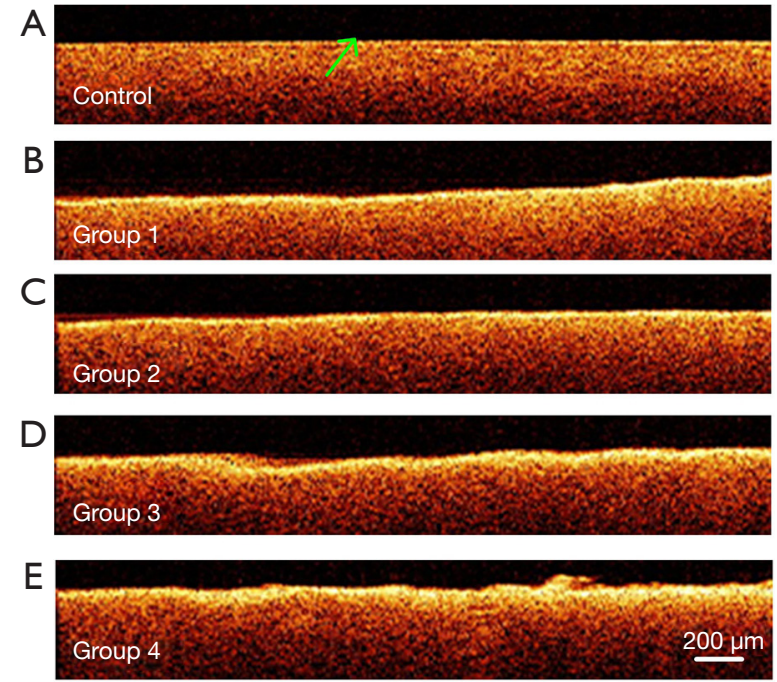

Figure 8 The OCT images of bone tissues before (A) and after Er:YAG laser ablation (B,C,D,E) at four different moisture contents (Groups 1-4). Scale bar: $200 \mu \mathrm{m}$. The green arrow indicated the boundary between bone tissue and air. OCT, optical coherence tomography.

the fact that the pulse width $(100 \mu s)$ used in this study was close to $400 \mu$ s, residual heat under laser irradiation without water cooling could spread to the surrounding tissue, and thus further micro-explosion shock could cause microcracks in the tissue. In this study, the thermo-melting effect was obvious, and more cracks were observed in the SEM results of the dry groups and non-sprayed groups. Er:YAG laser ablation of hard tissue is based on the thermo-mechanical theory. Water absorbs the laser energy and then vaporizes rapidly, resulting in micro-explosion within a small range of laser irradiation. Thus, in this way, accurate cutting can be achieved, similar to blasting rock with explosives. The faster the laser pulse, the shorter the time it takes for heat to transfer to the surrounding tissue, minimizing lateral thermal damage (25).

The response of biological tissue to laser irradiation largely depends on the nature of its components. Bone is composed of apatites surrounded by an organic matrix. In this study, EDX spectroscopy results showed a clear increase of calcium and phosphorus content in Groups 2, 3 and 4, and that laser ablation with water spray does not affect the calcium and phosphorus content in undried bones. In a study by Sasaki et al., the chemical composition of laser-irradiating bone tissues in rat skull was analyzed by EDX spectroscopy, and the calcium, phosphorus 

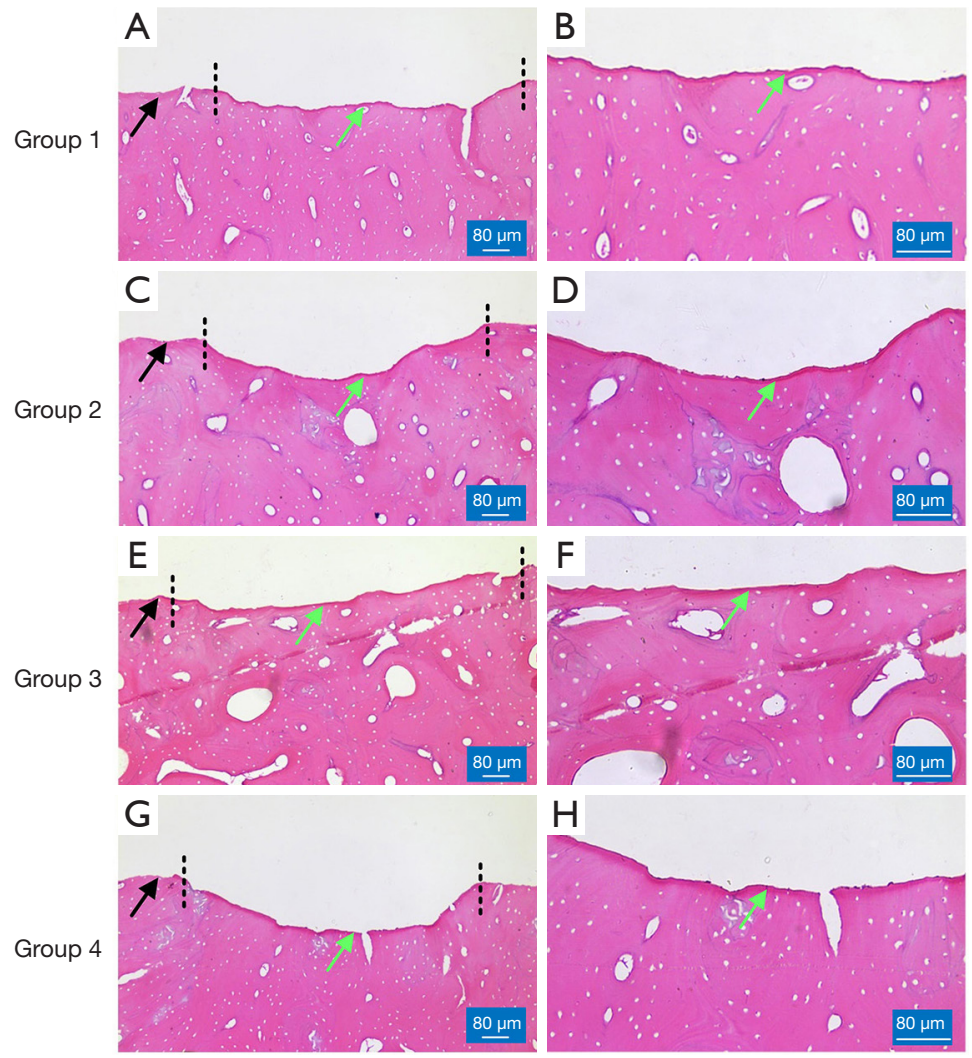

Figure 9 The histological sections of bone tissues after Er:YAG laser ablation. In Group 1 (A,B), Group 2 (C,D), Group 3 (E,F), and Group $4(\mathrm{G}, \mathrm{H})$. Scale bar: $80 \mu \mathrm{m}$. The green arrows indicate the deep stained layers. The black arrows indicate the non-irradiated areas. Dashed lines label the boundary of laser ablated areas.

content, and $\mathrm{Ca} / \mathrm{P}$ ratios were all found to be decreased (26). Nevertheless, Panduric et al. observed no differences in calcium, phosphorus content, or $\mathrm{Ca} / \mathrm{P}$ ratios between Er:YAG laser or low-speed guided drill-treated pig ribs (27). Differences in laser-irradiated parameters, sample types, and experimental design can explain the inconsistency in results $(28,29)$.

The OCT system has three advantages: first, it has high imaging resolution and can perform depth imaging; second, it has a large imaging range and fast scanning speed that enable real-time 3D imaging; and third, it is a noncontact and non-invasive imaging mode as the front end of OCT system can be miniaturized. Based on the advantages mentioned above, the OCT system has been tested for evaluating the effect of laser ablation of bone tissue in the past few years. Ohmi et al. and Zhan et al. measured the depth of craters induced by Nd: YAG laser or $\mathrm{CO}_{2}$ laser on the hard bone surface (30,31). More recently, Fuchs et al. achieved online measurement and evaluation of the
Er:YAG laser ablation process using an integrated OCT system (32). Previous studies have focused on the evaluation of ablation efficiency, explicitly measuring the depth of the ablation crater. OCT technology has developed rapidly in recent years. Our OCT system could image $0.5 \times 4 \times 2 \mathrm{~mm}^{3}$ bone tissue in 2 seconds. In this study, the highest ablation could be seen in dry bones without an external water spray (Group 4), as shown in Figure 7. The ablated depth was significantly lower in the groups with external water spray, which is consistent with the results of Meister et al. (33). On the contrary, different internal water volume had little effect on tissue ablation efficiency. This study demonstrated that real-time imaging of Er:YAG laser ablation grooves could be realized based on the 3D OCT system.

Based on its broad field view 3D imaging capacity, OCT could provide information about the ablation groove. In addition to visual evaluation, the OCT features highresolution imaging that allows the quantification of the roughness of the ablation groove surface. Five surface lines 

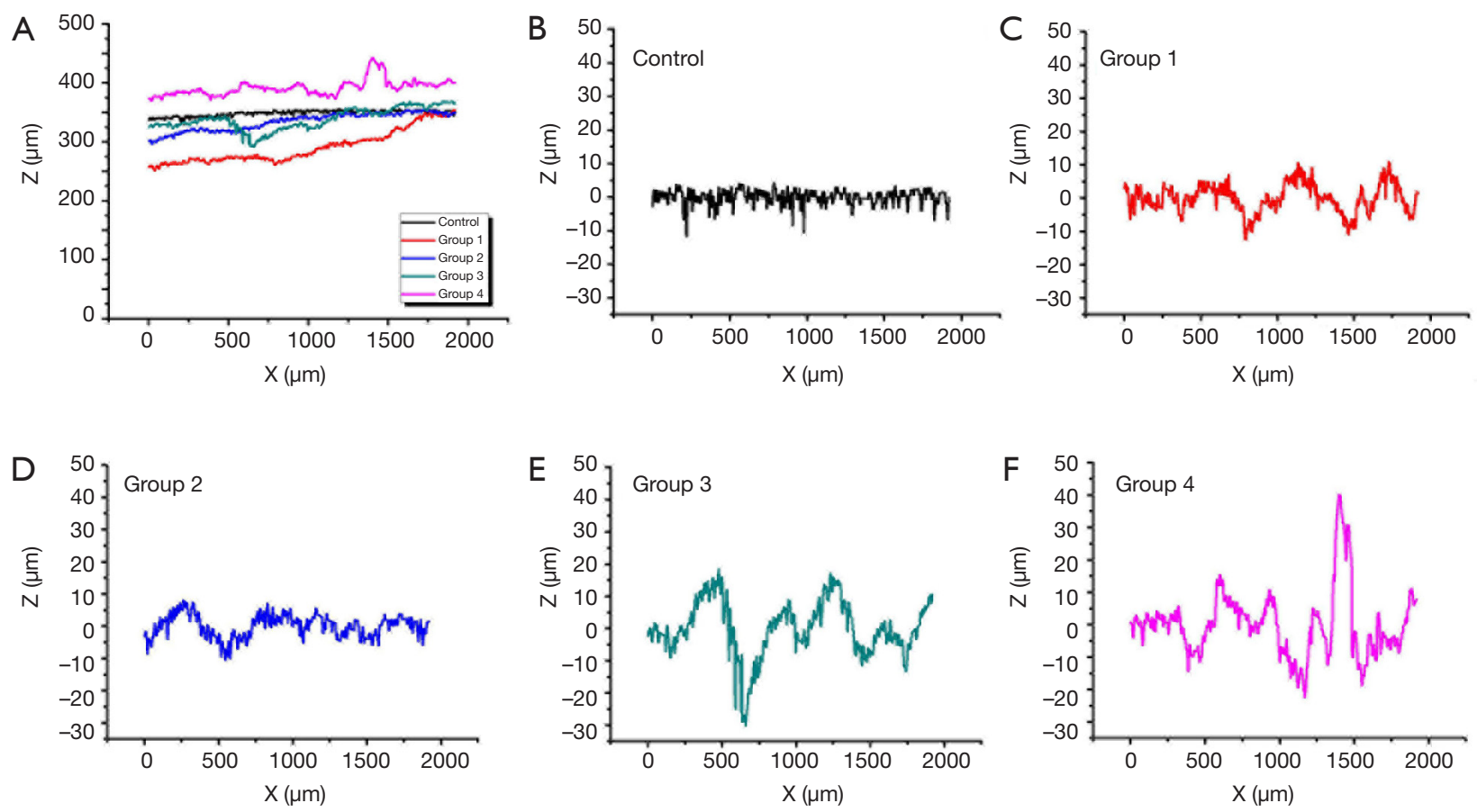

Figure 10 Quantitative evaluation of surface roughness. (A) Surface lines extracted from the OCT images shown in Figure 8. (B) Calibrated surface line of the control group without Er:YAG laser ablation. (C,D,E,F) showed the calibrated surface lines of bone ablated with Er:YAG laser at four different moisture contents (Groups 1-4). OCT, optical coherence tomography.

were extracted from the OCT images, as shown in Figure 8. The original surface lines with $2 \mathrm{~mm}$ length are shown in Figure 10A. For a clear illustration of surface roughness, baseline calibration was performed on each surface line. Figure 10B,C,D,E,F provide five calibrated surface lines. Obviously, the surface line of the control group was the flattest, followed by the surface lines of Groups 1 and 2 . The surface lines of Groups 3 and 4 were the most rugged. Moreover, similar roughness was observed between Groups 1 and 2, as well as between Groups 3 and 4. This confirms the fact that water spray plays an important role in the roughness of bone surface induced by Er:YAG laser ablation.

Furthermore, our OCT system could achieve depth imaging of bone tissue with a thickness of over 400 microns. This imaging capability is beyond the capacity of other existing imaging technologies. Based on the high-resolution cross-sectional OCT image of the bone tissue surface, it could be clearly found that the boundary between the bone tissue surface and air in the control group was straight, and the bone under the decomposition line was very uniform. After Er:YAG laser ablation, the surface of the bone tissue was uneven, and there were distinct bright layers on the surface. Quantitative evaluation can be realized by the extraction and analysis of image intensity. Image intensity profiles (with $200 \mu \mathrm{m}$ length) covering the boundary and bright layers were extracted from the OCT images (control group, Groups 1-4), as shown in Figure 11. The maximum value of each image intensity profile was different but insignificant. The FWHM was measured and marked on each line as a quantitative evaluation standard. These layers in the non-sprayed groups were thicker than that in the control group and water spray groups, indicating that the thickness of heterogeneous layers had a positive correlation with the corresponding thermal damage of the laserirradiating tissues. The water content in cortical bone does not affect the thickness of heterogeneous layers after laser ablation. On the contrary, external water supply makes it possible to reduce the thermal damage to the surrounding tissues.

Similarly, thin eosin-stained layers were observed in all the experimental groups through histology. The eosinstained layers in the non-sprayed groups were thicker than those in the non-irradiating areas and water spray groups. This is consistent with the results of Yoshino et al. (34), who used Er:YAG laser (pulse energy $115 \mathrm{~mJ}$, pulse 


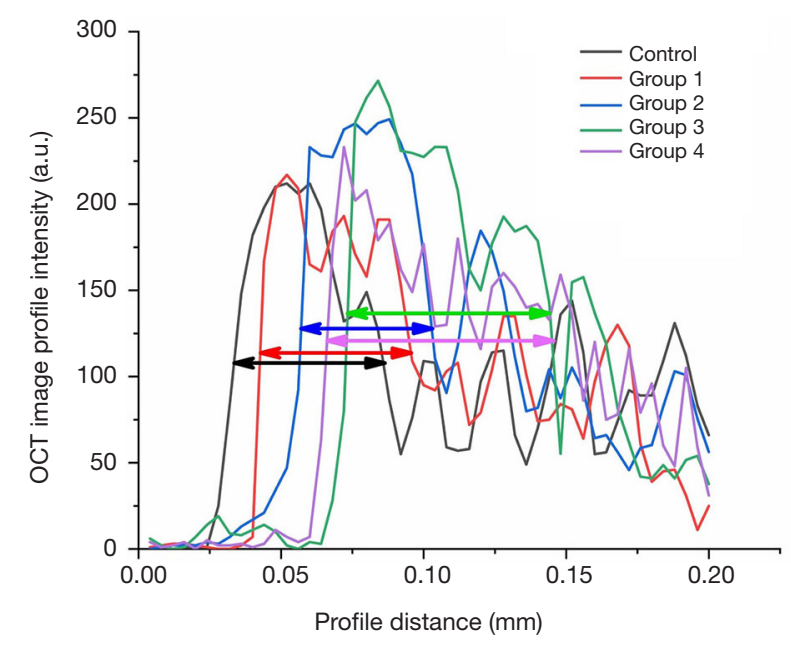

Figure 11 The OCT image profile intensity of bone before (control group) and after Er:YAG laser ablation at four different moisture contents (Groups 1-4), as shown in Figure 8. The length of the two-way arrow line is the full width at half maximum (FWHM) of the corresponding OCT image profile intensity line. OCT, optical coherence tomography.

repetition frequency $10 \mathrm{~Hz}$ ) without water spray to ablate the cortical bone of rat skull and observed a thin denatured layer without serious thermal damage by histology. It is also worth noting that there was good agreement between the denatured layers obtained by OCT and histology.

In this study, four different moisture content models were constructed by changing internal water content and external water spray. Compared with normal bone, the irradiated bone with high water content and external water spray showed no significant change of calcium and phosphorus, representing lower thermal damage. This study had some limitations that need to be addressed, such as bone samples and Er:YAG laser parameters should be more diversified. However, we observed that OCT allows the real-time imaging and assessment of alterations of surface properties of cortical bone after Er:YAG laser irradiation at different moisture contents without having to harvest specimens of tissue. Large field of view 3D OCT imaging could be used to evaluate ablation efficiency and ablation groove morphology. Surface lines could be extracted from high-resolution OCT images and be used to evaluate roughness. OCT images with large imaging depth realized non-destructive visualization of a heterogeneous layer on cortical bone surface induced by Er:YAG laser ablation. This study demonstrated that different moisture contents led to different ablation effects, and OCT could quickly and accurately evaluate the differences between them, as compared to histology and SEM. In conclusion, this study will promote the application of OCT technology in the real-time evaluation of Er:YAG laser ablation of bone.

\section{Acknowledgments}

Funding: This research is supported by the National Natural Science Foundation of China (No. 11704082 and 81470724), Start-up grant from Guangzhou Medical University, Guangzhou Science, Technology, and Innovation Commission (No. 201704030024), Natural Science Foundation of Guangdong Province (2020A151501118).

\section{Footnote}

Conflicts of Interest: The authors have no conflicts of interest to declare.

Open Access Statement: This is an Open Access article distributed in accordance with the Creative Commons Attribution-NonCommercial-NoDerivs 4.0 International License (CC BY-NC-ND 4.0), which permits the noncommercial replication and distribution of the article with the strict proviso that no changes or edits are made and the original work is properly cited (including links to both the formal publication through the relevant DOI and the license). See: https://creativecommons.org/licenses/by-nc-nd/4.0/.

\section{References}

1. Rupprecht $\mathrm{S}$, Tangermann K, Kessler P, Neukam FW, Wiltfang J. Er:YAG laser osteotomy directed by sensor controlled systems. J Craniomaxillofac Surg 2003;31:337-42.

2. Moosavi H, Ghorbanzadeh S, Ahrari F. Structural and Morphological Changes in Human Dentin after Ablative and Subablative Er:YAG Laser Irradiation. J Lasers Med Sci 2016;7:86-91.

3. Li T, Zhang X, Shi H, Ma Z, Lv B, Xie M. Er:YAG laser application in caries removal and cavity preparation in children: a meta-analysis. Lasers Med Sci 2019;34:273-80.

4. Hibst R, Keller U. Experimental studies of the application of the Er:YAG laser on dental hard substances: I. Measurement of the ablation rate. Lasers Surg Med 1989;9:338-44.

5. Contreras-Arriaga B, Rodríguez-Vilchis LE, ContrerasBulnes R, Olea-Mejìa OF, Scougall-Vilchis RJ, Centeno- 
Pedraza C. Chemical and morphological changes in human dentin after Er:YAGlaser irradiation: EDS and SEM analysis. Microsc Res Tech 2015;78:1019-25.

6. Bader C, Krejci I. Indications and limitations of Er:YAG laser applications in dentistry. Am J Dent 2006;19:178-86.

7. Evans DJ, Matthews S, Pitts NB, Longbottom C, Nugent ZJ. A clinical evaluation of an Erbium:YAG laser for dental cavity preparation. Brit Dent J 2000;188:677-9.

8. Grzech-Leśniak K, Matys J, Żmuda-Stawowiak D, Mroczka K, Dominiak M, Brugnera Junior A, Gruber R, Romanos GE, Sculean A. Er:YAG laser for metal and ceramic bracket debonding: an in vitro study on intrapulpal temperature, SEM, and EDS analysis. Photomed Laser Surg 2018;36:595-600.

9. Kesler G, Shvero DK, Tov YS, Romanos G. Platelet derived growth factor secretion and bone healing after Er:YAG laser bone irradiation. J Oral Implantol 2011;37:195-204.

10. He Z, Chen L, Shimada Y, Otsuki M, Tagami J, Ruan S. Mechanical properties and molecular structure analysis of subsurface dentin after Er:YAG laser irradiation. J Mech Behav Biomed Mater 2017;74:274-82.

11. Trevelin LT, Silva BTF, Arana-Chavez VE, Matos AB. Impact of Er:YAG laser pulse duration on ultra-structure of dentin collagen fibrils. Laser Dent Sci 2018;2:73-9.

12. Pourzarandian A, Watanabe H, Aoki A, Ichinose S, Sasaki KM, Nitta H, Ishikawa I. Histological and TEM examination of early stages of bone healing after Er:YAG laser irradiation. Photomed Laser Surg 2004;22:342-50.

13. Zhang J, Ge W, Yuan Z. In vivo three-dimensional characterization of the adult zebrafish brain using a 1325 nm spectral-domain optical coherence tomography system with the 27 frame/s video rate. Biomed Opt Express 2015;6:3932-40.

14. Zhang J, Liu J, Wang LM, Li ZY, Yuan Z. Retroreflectivetype Janus microspheres as a novel contrast agent for enhanced optical coherence tomography. J Biophotonics 2017;10:878-86.

15. Luca RE, Todea CD, Duma VF, Bradu A, Podoleanu AG. Quantitative assessment of rat bone regeneration using complex master-slave optical coherence tomography. Quant Imaging Med Surg 2019;9:782-98.

16. Dao Luong MN, Shimada Y, Turkistani A, Tagami J, Sumi Y, Sadr A. Fractography of interface after microtensile bond strength test using swept-source optical coherence tomography. Dent Mater 2016;32:862-9.

17. Ding J, Ebihara A, Watanabe S, Iino Y, Kokuzawa C, Anjo T, Suda H, Sumi Y. Application of optical coherence tomography to identify pulp exposure during access cavity preparation using an Er:YAG laser. Photomed Laser Surg 2014;32:356-9.

18. Zhou Y, Shimada Y, Matin K, Sadrc A, Sumid Y, Tagami J. Assessment of bacterial demineralization around composite restorations using swept-source optical coherence tomography (SS-OCT). Dent Mater 2016;32:1177-88.

19. Genina EA, Bashkatov AN, Tuchin VV. Tissue optical immersion clearing. Expert Rev Med Devices 2010;7:825-42.

20. Zhou Z, Zhang Q, Wu W, Lin YH, Tai DI, Tseng JH, Lin YR, Wu S, Tsui PH. Hepatic steatosis assessment using ultrasound homodyned-K parametric imaging: the effects of estimators. Quant Imaging Med Surg 2019;9:1932-47.

21. Moshiri Y, Legocki AT, Zhou K, Cabrera MT, Rezaei KA, Tarczy-Hornoch K, Wang RK. Handheld sweptsource optical coherence tomography with angiography in awake premature neonates. Quant Imaging Med Surg 2019;9:1495-502.

22. Shu X, Beckmann L, Wang Y, Rubinoff I, Lucy K, Ishikawa H, Wollstein G, Fawzi AA, Schuman JS, Kuranov RV, Zhang HF. Designing visible-light optical coherence tomography towards clinics. Quant Imaging Med Surg 2019;9:769-81.

23. Lin Y, Xiang X, Chen T, Gao C, Fu H, Wang L, Deng L, Zeng L, Zhang J. In vivo monitoring and high-resolution characterizing of the prednisolone-induced osteoporotic process on adult zebrafish by optical coherence tomography. Biomed Opt Express 2019;10:1184-95.

24. Holmes JE, Bydder GM. MR imaging with ultrashort TE (UTE) pulse sequences: Basic principles. Radiography 2005;11:163-74.

25. Cersosimo MC, Matos AB, Couto RS, Marques MM, de Freitas PM. Short-pulse Er:YAG laser increases bond strength of composite resin to sound and eroded dentin. J Biomed Opt 2016;21:48001.

26. Sasaki KM, Akira A, Shizuko I, Isao I. Ultrastructural analysis of bone tissue irradiated by Er:YAG laser. Lasers Surg Med 2002;31:322-32.

27. Panduric DG, Juric IB, Music S, Molčanov K, Sušic M, Anic I. Morphological and ultrastructural comparative analysis of bone tissue after Er:YAG laser and surgical drill osteotomy. Photomed Laser Surg 2014;32:401-8.

28. Jung MK, Kim SG, Oh JS, Jin SC, Lee SY, Jang ES, Piao ZG, Lim CS, Jeong MA. A comparative histological study of bone healing in rat calvarial defect using the erbium-doped yttrium aluminum garnet laser and rotary instruments. Jpn J Appl Phys 2013;51:34-6. 
29. Daskalova A, Bashir S, Husinsky W. Morphology of ablation craters generated by ultra-short laser pulses in dentin surfaces: AFM and ESEM evaluation. Appl Surf Sci 2010;257:1119-24.

30. Ohmi M, Wa M, Fukunaga A, Haruna M. In-situ observation of tissue laser ablation using optical coherence tomography. Opt Quant Electron 2005;37:1175-83.

31. Zhan Z, Zhang X, Ye Q, Xie S. Measurement of crater geometries after laser ablation of bone tissue with optical coherence tomography. Chin Opt Lett 2008;6:896-8.

32. Fuchs A, Schultz M, Krüger A, Kundrat D, Diaz JD, Ortmarier T. Online measurement and evaluation of the

Cite this article as: Huang W, Gao C, Lan Y, Zeng S, Pathak JL, Zhou M, Ge L, Zhang J. Optical coherence tomography characterizes the roughness and thickness of the heterogeneous layer on cortical bone surface induced by Er:YAG laser ablation at different moisture contents. Quant Imaging Med Surg 2020;10(3):713-726. doi: 10.21037/qims.2020.02.15
Er:YAG laser ablation process using an integrated OCT system. Biomed Eng 2012;57:434-7.

33. Meister J, Franzen R, Forner K, Grebe H. Influence of the water content in dental enamel and dentin on ablation with erbium YAG and erbium YSGG lasers. J Biomed Opt 2006;11:34030.

34. Yoshino T, Aoki A, Oda S, Takasaki AA, Mizutani K, Sasaki KM, Kinoshita A, Watanabe H, Ishikawa I, Izumi Y. Long-term histologic analysis of bone tissue alteration and healing following Er:YAG laser irradiation compared to electrosurgery. J Periodontol 2009;80:82-92. 